\title{
SUMMARY OF THE WORKSHOP ON PERINATAL AND POSTNATAL DEFECTS AND NEUROLOGIC ABNORMALITIES FROM CHEMICAL EXPOSURES
}

\author{
Chairpersons: \\ Mason Barr, Jr. \\ University of Michigan School of Medicine \\ Ann Arbor, Michigan 48104 \\ Carl A. Keller \\ National Institute of Child Health and Human Development \\ National Institutes of Health \\ Bethesda, Maryland 20014 \\ Walter J. Rogan \\ National Institute of Environmental Health Sciences \\ National Institutes of Health \\ Research Triangle Park. North Carolina 27709 \\ Invited Participant: \\ Jennie Kline \\ Columbia University \\ School of Public Health \\ New York, New York 10027
}

\section{INTRODUCTION}

Both chemical pollution and human reproduction are complex topics. Inquiry into chemical-related damage to human reproduction is hampered by this complexity. In general, the whole area of environmental chemicals versus human reproduction is one of great concern but, at this time, there are few hard data. The following provides an analytic framework for discussion of these topics, and also provides an outline for this presentation.

1. Specific chemical substances that occur in the environment constitute exposures. These may be widespread or occur only in special circumstances.

2. The events of the reproductive process constitute outcomes. These will be ordered temporarily, together with some comment on feasibility of ascertainment.

3. Clinical examinations and tests, chemical analyses, and historical information constitute measures. These may be gathered routinely and easily available, or require specific special efforts. 
Opportunities for study occur when ascertainment of the above occur jointly, together with the investigator's suspicion of an etiologic relationship between exposure and damage. This presentation will explore each of the areas, with comments on the plausibility of etiologic relationships and feasibility of their study.

\section{Exposures}

Exposures to environmental chemicals occur in a number of ways. However, we will concentrate here on exposures that are chronic, such as in the workplace, or persistent, such as to chemicals that are not readily metabolized or excreted. Except as examples of mechanisms, we will not discuss short-term, acute exposures nor exposures to drugs. Exposures to environmental chemicals take place in one of the three following ways; there is, of course, some overlap for specific chemicals.

1. Occupational Exposure. This usually involves a rather well-defined group of workers, and sometimes their families and associates by home contamination.

2. Accidental Exposure. This occurs through a specific contamination incident, such as the PBB incident in Michigan' or the dioxin incident in Seveso, Italy. ${ }^{2}$ The population may be easily identifiable, but is generally less well defined and more difficult to follow than an occupational group.

3. Ambient Exposure. This occurs through media, such as air, water, and food, variously contaminated by environmental chemicals. Specific populations of exposed and unexposed individuals are often not available for study, and the clear establishment of etiologic relationships thus is limited to severe disease (i.e., lead encephalopathy).

\section{Occupational Exposures}

Occupational exposures to toxic chemicals include both exposures to unusually toxic chemicals and exceptionally heavy exposures to less toxic substances. Both of these possibilities provide opportunities for observing the effects of chemical exposure in a unique situation, i.e., one that makes it relatively easy to identify a population at risk. The major problem, however, is to identify those chemicals for which appropriate toxic effects are to be measured or tested. For those chemicals that we know to be teratogenic, the intention is usually to decrease the exposure to the reproductively active worker presumptively. Similar reasoning applies to those exposures such as lead ${ }^{3}$ that are known to produce fetal wastage. Substances that are suspect for producing adverse pregnancy outcomes also include carcinogens and mutagens. Since this group of chemicals is so large, it is difficult to know where to begin.

One approach is to measure outcomes such as congenital malformations and pregnancy wastage in certain employment situations where a significant proportion of workers are female, and where a number of chemicals are used. Examples would include nurses, dry cleaning workers, garment workers, and textile workers. Another approach is to select certain chemicals as being suspicious on the basis of animal studies, widespread usage, or the observations of an alert practitioner. Although not so systematic, the latter method has most often been responsible for discovering occupation-related exposures that induce serious adverse health effects. Examples of this approach would be the association of adverse pregnancy outcomes and exposure to lead, ${ }^{3}$ hexachlorophene, ${ }^{4}$ and polychlorinated biphenyls. ${ }^{5,9}$ With the current emphasis 
on reproductive outcome as a major health issue, and with the increasing participation of women in the workplace, additional chemical toxins in the workplace will likely be jdentified.

\section{Ambient Exposure}

Ambient exposure to toxic chemicals takes place in two ways; one is basically purposeful, although not necessarily to a known toxic substance, and the other is accidental.

Purposeful exposures include smoking, beverage alcohol consumption and the use of other consumer producers such as saccharin, cyclamates, or red dye number 2 . The major component of purposeful exposure is a behavioral one in which the risks of toxic exposure are either thought to be offset by benefits or are not known. In other words, the exposure, via ingestion or inhalation or absorption through the skin, is done with knowledge of the exposure itself but without an adequate assessment of the consequences to reproductive outcome (or other health effects). Assessment of the extent of exposure is generally accomplished by measurement of intake, such as how many cigarettes are smoked, how many soft drinks consumed, etc.

Accidental exposures include those in which the individual is not necessarily aware that he or she is being exposed to the toxic substance. These constitute a very long list and include pesticides, industrial chemicals, contaminants and food additives. Assessment of exposure often requires measurements of the chemical in tissues or fluids, such as fat, blood, hair, milk, etc.

1. Pesticides

Among the more common pesticide residues found in human fat tissue are DDT, ${ }^{6}$ heptachlor, dieldrin, and hexachlorobenzene. ${ }^{7}$ These chemicals tend to persist in the environment and can reach human beings via the ingestion of animal products. Some pesticides are ingested directly as residues on vegetable material or are absorbed through the skin by careless use of commercial preparations used as consumer products.

\section{Industrial Chemicals}

Probably the most common and best known industrial chemicals that constitute a significant ambient exposure to humans are the polyhalogenated biphenyls, particularly PCBs. ${ }^{8}$ These chemicals were used in the manufacture of a number of products, especially electrical transformers and electronic parts. Electrical equipment is eventually discarded, and the PCBs can become air or water pollutants. Paper products may be recycled, and the contained PCBs may become part of food packaging. Exposures have been detected throughout the U.S. through the analysis of fat and milk samples. In Japan, rice oil was contaminated by PCBs and other chemicals. Consumers of the contaminated oil developed chloracne, the characteristic skin lesion of chlorinated hydrocarbon exposure. Children born to exposed mothers were small for dates and had pigmented skin. ${ }^{9}$ Administration of PCBs to pregnant monkeys have produced similar problems with infertility, fetal loss, and low birth weight ${ }^{10}$ The polyhalogenated biphenyls as a group have varying degrees of toxicity depending on the number and position of the halogen substitutions. "Since most of the industrial preparations are mixtures, this presents some problems in determining the potential toxicity of various compounds and for interpreting experimental and laboratory data.

Another source of exposure to toxic chemicals is through contamination during the manufacturing of other industrial chemicals. Examples of contaminated products that might have adverse effects on reproductive outcome include the furans in PCBs, 
dioxins in herbicides and other chemicals, chlorinated naphthalenes, chloroform and carbon tetrachloride. The latter two have been found in water supplies and pose an additional potential problem through reuse via recharging ground water with treated waste water. $^{12}$

Other sources of air and water contamination by the disposal of waste effluents from industrial installations can involve almost any chemical. Humans may be exposed through direct use of contaminated water and air as well as indirectly through the consumption of plant and animal products which have been, in turn, contaminated through air or water. This latter route is of particular concern for those chemicals, particularly the halogenated hydrocarbons, which are capable of biomagnification in the food chain.

Another ambient source of human exposure to chemicals capable of inducing adverse reproductive outcomes is the addition of various substances to products used for human consumption. The indirect "food additives" include diethylstilbesterol used to increase weight gains in feedlot cattle, antibiotic residues (particularly the tetracyclines) used as animal food additives to provide vitamins and reduce diarrheal disease, and lead used in pipes for transmitting water supplies.

In addition to the variety of substances and the various routes of human exposure, there are some chemicals that can and do enter the human body through multiple routes. As an example, and by way of summarizing the various sources of exposure, the case of lead contamination will be considered.

Lead is known to cross the placenta ${ }^{13}$ and is thought to induce fetal neurologic damage at blood levels that are not associated with encephalopathy. ${ }^{14.15}$ It is stable in both the body and the environment and is a widely used material. It has had a number of different applications over a long period of time.

Lead can be absorbed by ingestion and/or inhalation in certain manufacturing situations, including battery making and smelting. It can be carried home on clothes ${ }^{16}$ and this causes secondary as well as direct occupational exposure. It is found as an ambient air pollutant near motorways and refineries from its use in gasoline. It has constituted an inadvertant source of poisoning from ingestion of paint on old buildings, and to a much lesser extent, by its use in modern copper tubing joined with solder lead containing. It has been found in the past in canned milk and baby formula. Human exposure to lead can, thus, be either occupational or ambient. It can be a contaminant and a "food additive." Exposure can be either purposeful (albeit by young children, primarily) or accidental.

It should be noted that most of the current knowledge about the adverse reproductive effects of chemicals in the environment has come through the study of occupational groups or as a result of large scale accidental exposures, particularly those in which the effects were, or are, rather dramatic. There remains much to be learned about the effect of low-level exposure and subtle effects.

\section{OUTCOMES}

Classically, a teratogenic agent is one that induces structural malformations during the gestational stage of development. Recently, the definition of teratogenesis has been expanded to include aberrations of structure, growth and function, whether caused by genetically determined factors, by environmental factors, or by an interaction of genetic and environmental factors. Clearly, a death during the embryonic, fetal, or neonatal periods might be the result of aberrant prenatal development produced by a teratogenic agent, and thus, all pregnancy losses should be considered in the investigation of an environmental exposure. In like manner, 
alterations in fertility due to chemical agents might be classed as teratogenic effects. And finally, effects of exposures during development that lead to behavioral problems or physical disease such as cancer should be included. Thus, the complete spectrum of adverse reproductive outcomes which might be related to exposure to toxic agents can range from decreased fertility through fetal loss, growth retardation, and congenital malformations to childhood or even adult morbidity.

\section{Fertility}

Although fertility is measured as the efficiency with which a generation of people reproduces itself, it is more meaningful to divide this into components reflecting different stages in which adverse outcomes can occur as a result of exposure to toxic chemicals.

The earliest events which reduce reproductive efficiency are those that lead to a failure of gametocytes to develop into viable gametes. This can occur in either the male or the female, but, since gametogenesis proceeds differently in male and female mammals, toxic insults can have different consequences. In the sexually mature male, primary gametocytes are continuously converted through meiotic division into gametes. A disruption in this process can result in a reduced number of viable gametes. Such an effect has been observed with $\mathrm{X}$-irradiation and with some industrial chemicals, a recent example being Kepone .

The fernale gametogenic process starts during fetal development and oogonia reach a maximum in number (approximately $5 \times 10^{6}$ ) during the fifth month of gestation. A million or so oogonia differentiate into primary oocytes of which only a few hundred will subsequently form mature ova capable of being fertilized. Since only a relative few of the potentially available primary oocytes develop further, generally one at a time, it is unlikely that total infertility induced by the destruction of all available oocytes will occur from sublethal exposures to toxic substances. Recent evidence does suggest that an earlier menopause may occur as a result of more rapid depletion of primary oocytes, and cigarette-smoking women have been observed to experience menopause at younger ages than nonsmoking women in at least one investigation. Mouse studies also indicate that in some circumstances oocytes may be destroyed by chemical toxins and result in permanent total infertility.

The biologic processes involved suggest that temporary primary infertility or subfertility is most likely to occur in males exposed to toxic chemicals. Furthermore, detection of nonviable or morphologically abnormal gametes is considerably easier in the male by direct examination. Reduction in fertility can certainly occur in either sex, although it is more difficult to detect as primary in the female unless there is disruption or termination of the menstrual cycle. The unaided death of the conceptus is classified by the time of occurrence into spontaneous abortion $(<20$ weeks) and stillbirth $(>20$ weeks $)$.

\section{Death}

Deaths occurring during the first trimester are spontaneous abortions and include any loss from failure of implantation of the blastocyst to the death and expulsion of a 12-week fetus. Unfortunately these are often unrecorded events, and if early enough, are almost undetectable. Special studies can and have been undertaken to measure the occurrence and condition of spontaneous abortions. Current studies have reported that approximately $30 \%$ of spontaneous abortions are chromosomally abnormal and 
anatomic abnormalities may be present in an even greater proportion of such abortions. It is known that maternal age and radiation are both associated with aneuploidy (an abnormal number of chromosomes), particularly trisomy (one extra chromosome). Since most aneuploid embryoes die rather early during fetal development, they are considerably more common in spontaneous abortions than in later fetal deaths or live births. Although this affords an opportunity for the early recognition of adverse reproductive outcome due to toxic exposures, tissue cultures are required, the cost is high, and considerable expertise is needed. In one current study, a single technician is able to handle about three cultures per week. The sensitivity of such a system is potentially very good, however, if a good collection and pregnancy monitoring system is available and if the type of abnormality, either chromosomal or anatomic, can be specified for a given exposure. Unfortunately, an additional problem with the examination of first trimester spontaneous abortions is that they are often degenerated by the time examination is possible, which hampers both the detection of morphologic anomalies and the successful culture of tissues.

Losses during the second trimester are much easier to examine since they are larger and their existence is more likely to be detected. From 20 weeks onward, there is a requirement that a fetal death certificate be filed in all vital statistic jurisdictions in the U.S. There is evidence, however, that many fetal losses go unreported in spite of these requirements, and few are examined in detail for the presence of abnormality. Third trimester fetal losses, or stillbirths, are probably fairly well reported in most sections of the country, but as with second trimester fetuses, are often not studied in any detail.

After a live birth, death may occur during the neonatal period, infancy or childhood. The vast majority of these deaths ordinarily occur within the month following birth, particularly in the first few days. While reporting is good for these events, there are many factors other than exposure to toxic substances which are known to be associated with infant and childhood mortality. Furthermore, these events are relatively rare and very large populations must be followed to detect differences in either stillbirth or infant and childhood mortality rates in differentially exposed groups. This difficulty may be in part offset by the fact that data are readily available.

\section{Malformation}

With some limitations on the collection of data, measurement of birth defects is a direct and useful means of assessing the potential toxic effects of teratogenic agents. Special examinations and a more reliable reporting system have been initiated in some parts of the U.S. and studies in this and other countries continue to verify that some agents are teratogenic.

Major structural malformations occur in approximately $3 \%$ of all live births in the U.S. and in Europe, and if added to those among abortions and stillbirths, they make up a significant proportion of all human conceptions. Furthermore, some of them have clearly been found to be the result of exposure to chemical toxins during fetal development, although the vast majority of birth defects at present are of unknown cause. One of the problems with measuring the rate of congenital malformations in different populations, however, is that, even though they are often obvious on examinations, they are frequently not recorded in routine record systems, i.e., birth certificates and fetal death certificates, and thus, require special reporting systems. It has also been well demonstrated that the detection of malformations is enhanced by repeated examination at intervals during infancy and early childhood. 


\section{Growlh}

One of the significant effects which can be due to exposure to toxic chemicals in utero is a decrease in growth rate of the developing fetus, and this effect may extend to postnatal development as well. Examples of two agents that have been implicated in fetal growth retardation are PCBs and cigarette smoking. PCB exposure in Japanese women consuming contaminated rice oil resulted in babies of lower than usual birthweight, and experiments with monkeys have led to similar results. ${ }^{17}$ In studies all over the world, cigarette smoking and exposure to chemical compounds found in cigarette smoke have been shown to reduce birth weight in human babies by about 200 grams not attributable to premature delivery. Furthermore, there is some evidence that growth rates of children born to smoking mothers are subnormal as late as 8 years later.

One of the attractive features of an effect on growth rate is that it is relatively easy to measure, since birthweight is almost universally recorded on birth certificates. This should be a good measurement for comparison of variously exposed groups, but, as with perinatal deaths, there are other factors such as altitude, race, and maternal nutrition which can influence birthweight. The value of birthweight data would be considerably enhanced by the simultaneous recording of the length of gestation, the crown-heel length and the head circumference.

\section{Functional Defects}

The largest class of effects that can be due to exposure to toxic agents in utero are those which adversly affect normal functioning. These may also constitute the most numerous problems associated with such exposure since they comprise biochemical, behavioral, and other defects, many of which are uncataloged.

1. Biochemical defects include abnormal functioning in both endocrine and enzymatic systems. Many of these are the result of genetic abnormalities and of ten require special test systems to detect the nature of the functional deficit. The systems themselves are interdependent and a number of subtle changes can occur that escape detection.

2. Behavioral defects are probably the least well understood of all congenital abnormalities from any cause. Their complexity matches that of the biochemical alterations, from which they may not always be separated. The most notable behavioral defect associated with toxic exposure in utero is the mental deficiency among children born around Minamata Bay in Japan. This exposure was due to organic mercury that accrued in fishery products from industrial wastes containing mercurial salts, and was first noted among local cats who became ataxic after eating shellfish from the Bay. The condition in human babies manifested itself as permanent, severe mental retardation, and is still a problem more than 20 years later.

Another more recently "discovered" behavioral defect due to intrauterine exposure is the fetal alcohol syndrome. In this case mental retardation has been found among babies born to women who were exceptionally heavy drinkers during pregnancy. ${ }^{18}$ The implications for an effect in not-so-heavy drinkers is currently being studied and it has been suggested that binge drinking among moderate drinkers may have teratogenic effects in some cases.

A subject of some current interest is the role of environmental toxins in producing hyperactivity in children. Naturally occurring salycilates and food additives that might induce hyperactivity have been investigated following 
reports by Feingold. Since this association is hypothetical, treatment with special diets was undertaken to see if a reduction of hyperactivity could be accomplished by feeding foods that did not include these presumably toxic substances. The apparent success of this treatment regimen led to the publicizing of this association in a book and was followed by many studies. These studies indicate that the presumed relationship between hyperactivity and food additives is not well enough understood. In addition, hyperactivity itself is a very difficult condition to measure with confidence.

Another association between hyperactivity and a toxic substance has been suggested from low-level, chronic lead intoxication. The question here, however, was which comes first, the hyperactivity or the lead ingestion due to pica appetite. A group of children who were classed as hyperactive were measured for blood lead concentration. Those for whom a presumed non-lead-related cause of hyperactivity could be ascertained had the same blood lead levels as normally active children. The group without known cause for hyperactivity had an increased lead burden, which implies that the hyperactivity did not cause the increased ingestion of lead.

Although both these relationships between toxic substances and hyperactivity are presumably due to ingestion of toxic substances after birth, they raise the possibility that some association may be due to exposures in utero.

The major problem with attributing behavioral effects to any cause is the measurement of behavioral effects, per se. Several scales have been used in addition to the Stanford-Binet IQ scale and its derivatives (which are only applicable to older children). Among the scales which have been developed for infants are the following:

Apgar Score: This score is usually computed at one minute and five minutes after birth and is a measure of the general condition of the infant. It is based on a simple assessment of the normality of the heart rate, respiratory effort, muscle tone, reflex irritability and color, and it is moderately predictive of future neurologic status.

Prechtl Neurologic Examination: This is an assessment of neurologic adequacy for newborn babies and requires special training for its administration and evaluation.

Brazelton Neonatal Behavioral Assessment: This is a well-developed scale intended as a means of measuring interactive behavior. Although it contains a few neurologic items, its main thrust is behavioral and is an attempt to score the infant's available responses to his environment. The examination is usually performed on the third or fourth day of life and scoring is based on the infant's best performance rather than on an average response. The examination has shown good reliability when repeated at one month of age.

Bayley Scale: This scale is an assessment of gross motor, fine motor, language, and social functioning in older infants. It is in many ways comparable to an IQ scale, but is not always a reliable predictor of standard IQs measured at older ages.

There are a number of other scales that could be used and special procedures have been developed for specified problems. One advantage of the Apgar Score, in spite of its very limited scope, is that it is very fast, is routinely recorded following most deliveries in the U.S., and is often included on birth certificates. All of the others require somewhat elaborate examinations and 
have mostly been limited to special research protocols. Thus, while of undoubted importance in evaluating risks due to intrauterine exposures to potentially toxic substances, the assessment of functional behavioral defects is a difficult task.

3. Immunologic defects due to toxic exposure are just beginning to be investigated. There is some evidence that polybrominated biphenyls may reduce immunologic response in adults and similar findings among newborns could lead to more research in this area. Laboratory procedures to test immunologic competence are complex and probably not well enough developed at present for large scale studies, however.

To complete the spectrum of functional defects which could be due to teratogenic agents, one should include physical defects. These are discussed as malformations, although there is clearly overlap between anatomic malformations and functional defects.

\section{Pharmacologic}

A final group of infant health problems that can be included under adverse reproductive outcomes are those in which the toxic substance has crossed the placenta and produced a relatively pure pharamacologic response in the fetus. A prime example of this is heroin, in which the newborn infant suffers withdrawal symptoms similar to older persons who stop using the drug. Other examples would include drugs used for medical or psychotropic purposes, but such drugs have not been included in the present discussion of environmental exposures.

\section{The Occurrence of Adverse Reproductive Outcomes as Related TO ENVIRONMENTAL TOXIC EXPOSURES}

At the present time, at least $5 \%$ of all infants born alive in the U.S. either have a serious disease, a significant anatomic malformation, or a severe functional defect or die soon after birth. Estimates of the causes of these adverse outcomes have been made as follows ${ }^{19}$ :

$\begin{array}{lr}\text { Genetic factors } & 20 \% \\ \text { Infections } & 2-3 \% \\ \text { Maternal conditions } & 1-2 \% \\ \text { Environmental exposures } & 2-3 \% \\ \text { Chromosomal aberrations } & 3-5 \% \\ \text { Unknown } & 65-70 \%\end{array}$

As can be seen, the ascribed environmental causes account for only a small fraction of these, and this includes exposure to nonchemical agents such as radiation and trauma. An undetermined portion of the unknown causes, and some of the genetic and chromosomal causes, may be the result of toxic exposure in utero or to the gametocytes. Considering the large number of spontaneous abortions and the undetermined amount of infertility or subfertility that may exist in the population, plus the functional behavioral deficits not detectable until children are older, the possibilities for adverse reproductive outcomes which might be caused by chemicals that are toxic to the reproductive system or its products are large.

One way of modeling the various outcomes and their relation to toxic exposure is 
by use of a graded dose-response relationship. Such a model would appear as in FIGURE 1, and would include most of the outcomes that have been discussed.

This model suggests that a given toxic substance could lead to several different outcomes with different incidence rates at the same dose as well as show a dose-response increase in effect for any one outcome. This is consistent with the concept that almost any chemical is toxic to almost every system at some dose. The ordering of the different adverse reproductive outcomes might vary somewhat with different chemical toxins, and the slopes of the dose-response curves would be expected to vary as well.

The advantage of viewing the relationship between toxic chemical exposures and adverse reproductive outcomes according to the above model is that for any toxic chemical, certain outcomes are likely to be more sensitive indicators of effects. Thus, it becomes important to try to select the specific outcome that is most sensitive when assessing the effects of toxic exposure. For example, comparing the rates of all malformations may not reveal differences between exposed and nonexposed populations, while rates for a specific malformation, such as cleft lip/palate or digital hypoplasia, may do so in the case of the fetal hydantoin syndrome ${ }^{20} \mathrm{Or}$, in the case of smoking during pregnancy, there is considerable controversy over the effect on fetal loss, but intrauterine growth retardation has been a consistent finding.

Another important consideration in describing the relation between exposure and outcome is the interrelationship between various factors that effect the same outcome. This is termed confounding. For those confounding co-variables that are not of interest to the investigation of the teratogenic effects of toxic substances, an adjustment can often be made during analysis of data. An example of this is in measuring the effect of a teratogenic agent on chromosomal aberrations that increase with maternal age. If exposed women have higher rates of trisomy than nonexposed women at all ages, then the relationship between these factors is said to be independent and additive, and no interaction exists. There are situations, however, when the relationship is not additive, and an interaction does exist. If the combined effect of two (or more) agents on adverse reproductive outcome is greater than would be expected when each is acting alone and independently, then the agents are said to be synergistic; an example might be diphenylhydantoin and phenobarbital, which have been suspected of teratogenic synergism. ${ }^{21}$ If the combined effects are less than expected when either agent is acting

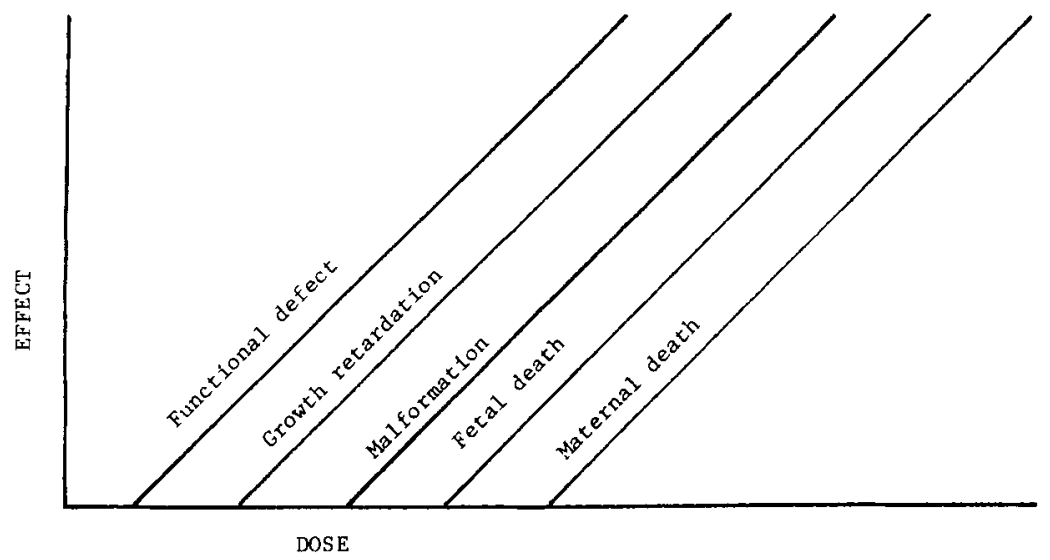

FIGURE 1. 
alone, they are said to be antagonistic, as in the case of phenobarbital protecting against the teratogenicity of cyclophosphamide. ${ }^{22}$ Thus, synergism between two or more factors may make it easier to detect teratogenic effects, but may also be misleading, while antagonistic effects may obsure the detection of teratogenic potential. Interactions between environmental agents involve more than chemicalchemical interactions, for it has been established that teratogenicity of various compounds can be modified by altering the oxygen supply to or the temperature of the fetus. ${ }^{22 A}$ And to complicate matters further, a vast literature on the great importance of gene-teratogen interaction has accumulated over the past several years. ${ }^{22 B}$

An additional consideration in assessing teratogenicity is the detection of anamnestic response. Such a response is due to a form of sensitization that can occur in utero and is a potential problem when measuring adverse outcomes following exposure to substances which mimic hormones and antigens. Early exposures to the fetus may alter subsequently occurring responses to otherwise benign agents.

This brief description of the range of relationship between toxic chemicals and their effects on human reproductive outcome should serve to assist in the design of studies and the interpretation of data on teratogenic potential.

\section{Measurement of the Association between Toxic Chemical Exposure AND AdVERSE REPRODUCTIVE OUTCOME}

In order to establish that an association exists between an exposure to any risk factor, such as a chemical toxin, and an adverse health outcome, such as congenital malformation, one of two general methods are employed. The prospective approach is to identify an exposed population and one that has not been exposed and then to examine them for the presence of a health condition possibly attributable to the exposure. A comparison of the rates of disease between the exposed and nonexposed populations will indicate the additional risks presumably due to the exposure.

The retrospective approach is to identify a group of individuals in which some adverse health outcome of interest has occurred, and a group of "healthy" individuals and determine their status vis-⿳亠㐅冋-vis past exposure to some agent. Both methods are equivalent for determining the relative increase (decrease) in disease that might be attributable to the exposure. In addition, in both situations a number of other factors can be taken into account and dose-response relationships can sometimes be established.

The advantage of the prospective method is that the time sequence of events, i.e., exposure before outcome, can be established unequivocally since exposure status is determined before knowledge of outcome status. Furthermore, during the course of such a study, the rate of occurrence of the outcome in the population being studied can be determined. If the outcome is rather rare, however, large populations may have to be followed and examined in order to make statistically reliable comparisons between exposed and nonexposed groups. The major advantage of the retrospective approach is that it is much more efficient in terms of time and resources, particularly for a rare disease outcome. A number of study designs using one or the other of these approaches have been developed, and others are possible.

The enormous number of potentially harmful chemicals and the wide variety of exposures plus the broad spectrum of adverse reproductive outcomes makes it impossible to measure all associations which might exist. Epidemiologic investigations, therefore, must attempt to concentrate on either the exposure or the outcome. The prospective method is generally used when exceptionally exposed populations can be identified, such as occupational groups or those living or working near an accidental 
introduction of a toxic chemical into the environment. The usual strategy is to select those chemicals having high teratogenic potential as determined from laboratory or chemical evidence or those chemicals that are structually similar to known toxins and for which some group of persons have received sufficient exposure for harmful effects to occur with a frequency high enough to be detectable. The rate of occurrence of adverse health effects among highly exposed individuals can be compared to those of the general population or to a group of unexposed controls. This has been fruitful in discovering disease-causing agents among occupational and accidentally exposed groups, but is not always relevant to those receiving ambient exposures at lower doses - often a much larger group. For example, while pesticide workers may show a decrease in reproductive efficiency due to subfertility caused by certain pesticide exposures, it is not possible to extrapolate the effects to the population as a whole, which will be exposed to much lower doses. Furthermore, occupational groups are by definition "employed," and employed persons may be "healthier" and perhaps react differently to many chemical compounds than unemployed persons.

Another and more direct way to measure exposure is through the laboratory determination of toxic chemical levels in human tissues and fluids, such as hair, blood, fat, urine, and milk. Lead and mercury exposures have been measured in this manner and individuals identified for further follow-up of health effects. These procedures are also quite useful in determining cumulative exposures to certain compounds that accumulate in body fat, such as pesticides, halogenated biphenyls, and other aromatic hydrocarbons. Both fat samples and breast milk have been subjected to laboratory analysis, and in conjunction with other information on the subjects from which these samples have been obtained, some groups of exposed individuals have been identified. Consumers of sport fish from fresh water sources in the U.S. have shown increased PCBs in breast milk and persons living in rural areas show higher levels of some pesticides than city dwellers.

The major usefulness of these studies, however, has been to identify areas of the country where exposures are high or low and to document long-term trends in general exposure. Recent data from the routine collection and analysis of fat samples and other materials from cadavers and biopsies across the U.S. by the Environmental Protection Agency has shown a decline in DDT in the 1970s following the curtailment of its domestic agricultural use. The national breast milk survey has identified areas in the East which have higher levels of PCBs in human breast milk than in the West. Both of these compounds are known or strongly suspected of being toxic to the reproductive system. The limited amount of material available for analysis makes it impossible to test for a large number of chemical compounds on a routine basis.

Thus, the prospective method of identifying and following up exposed groups has been most useful in verifying adverse reproductive or other detrimental health effects among heavily exposed occupational groups or those subjected to accidental dissemination of toxic chemicals. The method is most applicable in cases where some toxicity is known or is highly suspicious based on other evidence.

The retrospective method of epidemiologic investigation begins with the identification of cases and compares their history of previous exposure to a group of healthy controls. Most of the teratogenic agents known to produce human congenital malformations have been discovered by noting an exceptionally large number of some relatively unusual condition among a small group of children in a short span of time. How many cases among how many children and in how short a time are necessary to alert investigators that something unusual has occurred depends on the rarity of the condition and the proportion of cases which are attributable to the particular exposure. Fewer than 20 cases of scrotal cancer led Sir Percivall Pott to conclude that this was an occupational hazard to young chimney sweeps in England in the 
eighteenth century, while 86 cases of congenital cataract alerted Gregg to the teratogenic effects of Rubella virus infection during pregnancy in Australia. It has taken hundreds of cases to document some of the problems associated with intrauterine irradiation and literally thousands of low birthweight babies to establish the growth retarding effects of maternal cigarette smoking.

Even though most of the human teratogens have been detected through retrospective investigations of unusual congenital malformations, the reporting of these conditions remains remarkably incomplete. A number of major easily detectable anomalies such as anencephaly and spina bifida may not be recorded on birth certificates and are, thus, unavailable for investigation. The complete reporting of anomalies is elaborate and expensive, although the Center for Disease Control has established a surveillance system covering approximately one-fourth to one-third of all U.S. births and a more thorough reporting in one large city (Atlanta). Reporting of still births of at least 20 weeks gestation and of deaths to infants born alive is required in the United States, but there is no reporting of infertility, spontaneous abortions, or of childhood morbidity because it would be prohibitively expensive and extremely difficult to do so on a routine basis.

Potentially, there are routinely collected data that might be useful in assessing adverse reproductive outcome of some types among geographic areas, and by extension, to other special population groups. Data such as fetal and infant death rates could be monitored and, as recently suggested by Dobbins, birth spacing data obtainable on birth certificates in 40 of the states could be used as an indicator of fertility. Both subfertility and early abortion losses will result in longer time periods between successive live or still births. In addition, special surveys might be conducted using brief telephone interviews to identify populations with varying reproductive outcome histories.

A county study of all births and perinatal deaths has recently been initiated, and should be continued on a reasonably current basis. Areas with high perinatal loss or unusually long birth spacing could then be investigated for accuracy of reporting and for unusual local circumstances that might contribute to these outcomes. Preliminary analysis of early neonatal deaths in this manner indicates that those areas in the Northeast and Midwest where industrial pollution is noteworthy are not among the counties with significantly elevated rates. Early neonatal losses are significantly higher in the Appalachia and Rocky Mountains and in isolated areas across the country. This pattern is not similar to that observed for cancer mortality in adults, particularly for gastrointestinal cancers, which are concentrated in the Northeastern counties near industrial areas.

Thus, while retrospective methods have led to the identification of several important human teratogens, they will require a more complete reporting system to collect case material than has been available if their potential is to be fully realized. It is unfortunate that we have had to rely so heavily on the observations of alert practitioners.

\section{SUMMARY}

The spectrum of adverse reproductive outcomes that may be the result of toxic insult to human reproduction should be expanded to include fertility problems, through structural malformation, fetal growth abnormalities, and fetal loss to childhood morbidity and behavioral functional abnormality. The large number of chemical agents potentially responsible plus the many possible means of human exposure makes it imperative that a systematic way to detect associations be 
considered. An attempt to describe the relationship between exposures and outcomes, and their interactions, has been presented and includes the ordering of outcomes and dose-responses leading to various possible outcomes. The methods for studying these relationships include following up observed exposures to determine outcomes and tracing back observed outcomes to determine exposures. Both methods have been useful and both have some severe limitations as well. The job is enormous, but the opportunities are ubiquitous.

\section{REFERENCES}

1. KAY, K. 1977. Polybrominated biphenyls (PBB) environmental contamination in Michigan, 1973-1976. Environ. Res. 13(1): 74-93, February.

2. Fuller, J. G. 1977. The Poison that Fell From the Sky. Random House. New York.

3. ANGLE, C. R. \& M. S. MCINTIRE. 1964. Lead poisoning during pregnancy. Am. J. Dis. Child 108: 431-439.

4. KäLlÉN, B. 1978. Hexachlorophene teratogenicy in humans reputed. JAMA 240: 1585086 .

5. Hirayama, C. 1976. Clinical Aspects of PCB Poisoning. In PCB Poisoning and Pollution. Chapter 6, pp. 87-104. Academic Press. New York.

6. EPA. DDT-A review of scientific and economic aspects of the decision to ban its use as a pesticide. EPA 540/1-7-022.

7. Mes, J., D. S. Campbell, R. N. Robinson \& D. J. A. Davies. 1977. Polychlorinated biphenyls and organochlorine pesticide residues in adipose tissue of Canadians. Bull. Environ. Contam. Toxicol. 17(2): 196-203.

8. EPA National Conference on Polychlorinated Biphenyls. Conference proceedings. EPA 650/6-75-004.

9. Kuratsune, M., Y. Masuda \& J. Nagayama. Some recent findings concerning Yusho. EPA 560/6-75-004, 14-29

10. Allen, J. \& D. H. Norback. Pathobiological responses of primates to polychlorinated biphenyl exposure. EPA 560/6-75-004, 43-49.

11. MCKINNEY, J. Toxicology of selected symmetrical hexachlorobiphenyl isomers: Correlating biologic effects with chemical structures. EPA 560/6-75-004.

12. Adams, C. E., M. F. HaY \& C. Lutwak-ManN. 1961. The Action of Various Agents Upon The Rabbit Embryo. J. Embryol. Exp. Morphol. 9: 469-491.

13. Palmisona, P. A., R. C. Sneed \& G. Cassady. 1969. Untaxed whisky and fetal lead exposure. J. Pediatr. 75(5): 869-871.

14. David, O., J. Clark \& K. Voeller. 1972. Lead and hyperactivity. Lancet 1: 900-903, October 28.

15. Landrigan, P., E. L. Baker, R. G. Feldman, et al. 1976. Increased lead absorption with anemia and slowed nerve conduction in children near a lead smelter. J. Pediatr. 89(6): 904-910, 1976.

16. Baker, E. L., D. S. Folland, T. A. TAYlor, et al. 1977. Lead poisoning in children of lead workers. New Engl. J. Med. 296, February.

17. AlleN, J. R. \& D. A. BARsotti. 1976. The effects of transplacental and mammary movement of PCB's on infant rhesus monkeys. Toxicology 6: 311.

18. Clarren, S. K. \& D. W. Smith. 1978. The fetal alcohol syndrome. New Engl. J. Med. 298: $1063-1067$.

19. Wilson, J. J. 1973. Environment and Birth Defects. Academic Press. New York.

20. ElSHONE, J. \& J. H. M. VANECK. 1971. Aangeboren misvormingen met name gespleten lip met of zonder gespleten verhemlte, bijkinderen van moeders met epilepsie. Ned. Tijdochor Geneeskd. 115: 1371.

21. Barr, M., A. K. Poznaski \& R. D. Schmickel. 1974. Digital Hypoplasia and Anticohvulsants During Gestation: A teratogenic syndrome? J. Pediatr. 84: 254-256.

22. Gibson, J. E. \& B. A. BECKER. 1968. Effect of phenobarbital and SKF-525A on the teratogenicity of cyclophosphamide in mice. Teratology 1: 393-398. 
22a. Franklin, J. B., A. F. Goldfarb, R. Matsomoto \& R. L. Brent. 1963. Modification by progestational compounds of the teratogenic effect of uterine vascular clamping. Fertil. Steril. 14: 365-369.

BRENT, R. L., B. T. Bolden \& A. Weiss. 1968. The use of uterine vascular clamping in the pregnant rat to modify the embryotoxic effects of anti-cancer drugs. Cancer Res. 28: $2001-2006$.

EdWards, M. J. \& R. A. Wanner. 1977. Extremes of Temperature. In Handbook of Teratology. Vol. 1. Chapter 12. J. G. Wilson \& F. C. Fraser, Eds. Plenum Press. New York.

22b. Fraser, F. C. 1977. Interactions and multiple causes. In Handbook of Teratology. Vol. 1. Chapter 13. J. G. Wilson \& F. C. Fraser, Eds. Plenum Press. New York.

23. Brilliant, L. B., G. VAn Amburg, J. ISbister, et. al. 1978. Breast-milk monitoring to measure Michigan's contamination with polybrominated biphenyls. Lancet: 643-646. 